\title{
On the Technical and Social Structure of Community Networks
}

\author{
Leonardo Maccari \\ Department of Information Engineering and Computer Science, \\ University of Trento, Italy \\ \{leonardo.maccari\}edisi.unitn.it
}

\begin{abstract}
A Community Network is a bottom-up network created by a community of people with the goal of building a selfowned, self-managed communication infrastructure. Community Networks are blooming, they range from small ones (tens of nodes) to gigantic ones (tens of thousands of nodes), they are made primarily of wireless links but in some cases they mix wired and wireless technologies. People running a Community Network wish to have more independence and more control on the infrastructure compared to what commercial ISPs offer. Such networks can not be understood without studying the interaction between the social and the technical aspects, since both layers are tightly intertwined. This paper will show some properties of three community networks, indicating that they have some structural peculiarities. It will also show a socio-technical analysis using data coming from the public mailing list of one of the community, in order to highlight issues that the community needs to address to guarantee its sustainable growth.
\end{abstract}

\section{INTRODUCTION}

A Community Network $(\mathrm{CN})$ is a communication network set-up by a community of people with a bottom-up, participatory approach. It is primarily a wireless mesh network, extended in some cases with wired connections. While the concept of $\mathrm{CN}$ is not new [1] their development in the last period was remarkable, and today, $\mathrm{CN}$ represent an extremely interesting and timely research topic due to (among others) three factors ${ }^{1}$ : The first is that recent standards (such as IEEE $802.11 \mathrm{n} / \mathrm{ac}$ ) make it possible to realize high-capacity wireless links that can reach a length up to tens of $\mathrm{km}$. This allows to create networks that cover entire cities with excellent performances. Thus, the scalability and the protocols for mesh networks that were of high interest in the 2000s, today can be finally applied to real networks made of hundreds of nodes.

The second factor is that CNs have shown to be an effective way to bring connectivity in underserved areas, so they are a valuable instrument against digital divide. An outstanding case is the Guifi.net $\mathrm{CN}$ that was awarded by the European

This work was financed partially by the University of Trento under the grant "Wireless Community Net-works: A Novel Techno-Legal Approach" - Strategic Projects 2014, and partially by the European Commission, H2020ICT-2015 Programme, Grant Number 688768 'netCommons' (Network Infrastructure as Commons).

${ }^{1}$ Recently, various large research projects focussed on CNs: see the CONFINE, CLOMMUNITY, P2PValue and netCommons projects, respectively at www.confine-project.eu, www.clommunity-project.eu, www.p2pvalue.eu and www.netcommons.eu

ISBN 978-3-901882-83-8 @ 2016 IFIP
Commission with the 2015 European Broadband Awards ${ }^{2}$.

The third factor is that $\mathrm{CNs}$ are unique environments to experiment inter-disciplinary research, since they can be better interpreted combining different research methodologies. In a $\mathrm{CN}$ each network node corresponds to a person, a family, an association or a small business. The management of the network is collective so the social dynamics inside the community influence the technical choices about the network itself. It is crucial to understand the motivations that drive the communities and the social norms that regulate them if one wants to propose solutions that are not only technically sound but also compatible with the open and participatory nature of a $\mathrm{CN}$. Such a mix of social and technical aspects makes a $\mathrm{CN}$ an example of the emerging paradigm of the so-called "Internet of People" (IoP), because the whole network infrastructure is shaped by the behaviours of the individuals and their group decisions. It is thus very interesting to understand if this original organization leads to networks with different features compared to other networks that have been already studied.

This paper contributes to this discussion and will analyse the data available from three community networks: the FunkFeuer network in Wien and Graz, and the ninux.org network in Rome (abbreviated respectively as FFWien, FFGraz, ninux). The goal of the paper is to answer two questions:

- Is the evolution of the network graph different compared to other communication networks, such as scale-free networks?

- Given that the goal of the community is to build a distributed network with a de-centralized management, is the result close to the expectations of the community?

\section{Motivations, DATA-SET, BACKGROUND}

There is a large body of works that suggest that many network graphs, including the Internet, show a scale-free behaviour, that is, the distribution of the degree of the nodes follows a power-law function. A scale-free network presents a small number of densely connected hubs that strongly influence the behaviour of a communication network. Hubs guarantee that the average shortest path grows slowly with the number of nodes, which is a positive factor because it keeps the round-trip-time low. However, hubs are enormously more important than other nodes, which is a negative factor, because

\footnotetext{
${ }^{2}$ see https://ec.europa.eu/digital-agenda/en/news/five-projects-got-first-evereuropean-broadband-award
} 
they introduce points of failures. This paper uses data from the FFWien and FFGraz network to verify if this pattern can be observed also in CNs.

Another level of interpretation is given by the sociotechnical analysis of ninux, that gives insights on the sustainability of the network itself. Recent social analysis [2] have shown that community networks have strong political motivations: the construction of an independent, robust, decentralized network infrastructure. Ninux is not an exception [3]: Ninux participants have a critical opinion of ISPs and service providers motivated by the recent discussions about neutrality, privacy and forced disconnections. They identify the root cause of these problems in the centralization (both technical and administrative) of the networks and of the services, and for this reason they build their own decentralized network, managed with a peer-to-peer approach. Peer-to-peer organization is a key feature of ninux: since the mesh network works without introducing hierarchies and layers, the community tries to reflect this approach also in the social organization. Thus, the ninux community did not create a formal association, it does not assign formal responsibilities and does not have "roles" assigned to people. The discussions in the community are primarily carried on in the mailing lists and in weekly faceto-face meetings, and decisions are taken with a consensusbased method. This approach is shared with other CNs and it is original in the communication panorama.

It is legitimate to ask what is the degree of success of ninux and its overall sustainability. To answer, it is crucial to remember that the network exists because the participants, through their social interactions, cooperate to reach a common goal so the social networking layer is as important as the technological one. If the network is not technically sound, it will fail in bringing services to the people, but also if the community is not participated enough, there will be a lack of the social capital needed to maintain the infrastructure. The key observation is that in a $\mathrm{CN}$ the social network and the network infrastructure can be linked, since every node belongs to a person. The two layers of analysis can be explored with the same instruments to understand if there are cross-layer single points of failure that can mine the future growth of the network.

\section{A. The data-set}

The three networks use OLSR (Optimized Link-State Routing), a link-state routing protocol that makes it possible for each node to be aware of the whole network topology. The communities publish the network topology dumped by the OLSRd daemon, that can be used to analyse the network evolution. The topology recorded by the routing daemon can be misleading: in some cases a number of devices placed in the same physical location are attached to a wired switch and each of them runs a separate instance of the routing protocol. For OLSR they are different nodes but, in practice, they are not. To merge these cliques, another source of information is needed. More details about the networks and the merging technique are out of the scope of this paper, the interested

\begin{tabular}{c|c|c|c} 
& FFWien & FFGraz & ninux \\
\hline maximum recorded nodes & 235 & 126 & 140 \\
\hline maximum recorded links & 450 & 181 & 158 \\
\hline time series available & yes & yes & limited \\
\hline first dump & $2013-07-27$ & $2007-03-31$ & $2014-1-14$ \\
\hline last dump & $2014-02-15$ & $2016-02-21$ & $2014-1-20$ \\
\hline dump interval & weekly & monthly & every 5 min \\
\hline node ownership & no & no & yes \\
\hline mailing list & no & no & yes
\end{tabular}

TABLE I: The summary of the available data

reader can find details in the published source code ${ }^{3}$ and in previous works [4][5].

The FunkFeuer networks publish a long history of dumps, while for Ninux only the current state is available, plus data collected in a week-long monitoring realized in 2014 [5]. Tab. I reports a summary of the data used for this paper. In the rest of the paper the time based evolution of the network always refers to the FreiFunk networks, instead when the analysis is done on a single snapshot, the sample with the largest number of nodes for each network in considered.

For the ninux network, two other sources of information were accessed. The first is a database containing the mapping between the physical node and the ID of a person that owns it, the second is the archive of the mailing lists of the ninux community of Rome for the year 2014.

\section{B. Related Works}

CNs have been the subject of a series of research works in the past years that had the goal of analysing their topological features [6][4][5][7] their routing solutions [8][9] and their social and management aspects [10][11]. This paper performs a different analysis based on two original elements, the first is the analysis of the time-evolution of the networks, which helps understanding what was, and potentially what will be the evolution of the network. The second is the mixed social and technological analysis aimed at identifying single points of failure in the techno-social organization of the network.

\section{THE NETWORK GRAPHS AND THEIR EVOLUTION}

Fig. 1 Fig. 2 and Fig. 3 show the relative frequency of the degree distribution for the three networks, and the best-fit with a power-law function. A power-law degree distribution is normally observable in the central part of a distribution or in the right tail. In this case the size of the networks (hundreds of nodes, and maximum degree that ranges from 11 to 29) makes it statistically hard to identify a trend.

An alternative approach is to investigate if the network evolution follows a preferential attachment model, which would lead to a more evident scale-free behaviour with the growth of the network. The preferential attachment model describes the way in which new nodes are added to the network, and the entry points they connect to. In such model the rate $\Pi(k)$ with which a node with $k$ links acquires new links is a monotonically increasing function of $k$. Following a preferential attachment model is not a necessary condition to

\footnotetext{
${ }^{3}$ accessible via Git at github.com:leonardomaccari/
} 


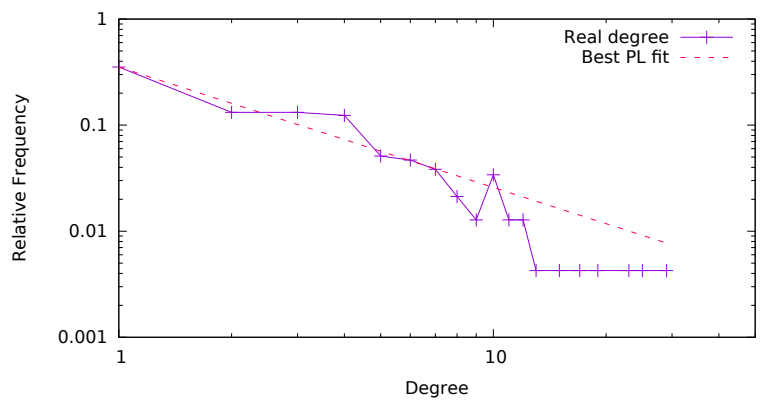

Fig. 1: The degree distribution for FFWien, and the best powerlaw fit $x^{-\alpha}, \alpha=1.13$.

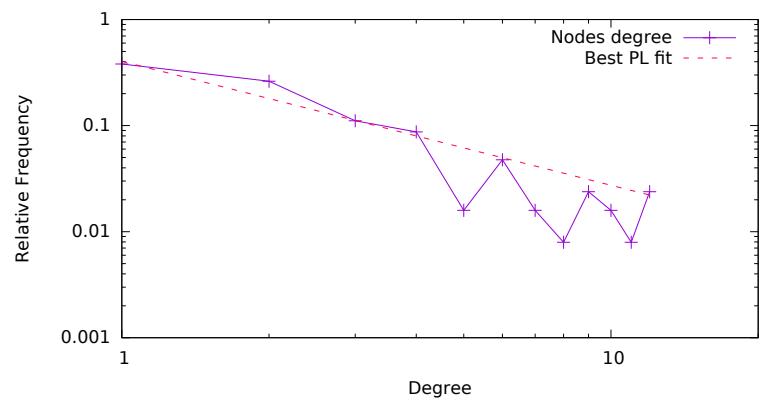

Fig. 2: The degree distribution for FFGraz, and the best powerlaw fit $x^{-\alpha}, \alpha=1.16$.

have a scale-free network, however since it has been shown that it is at the base of several different kinds of scale-free networks (the Internet graph for instance, has been shown to have $\Pi(k) \propto k$ so that the probability of acquiring now links is proportional to the current number of links of a node [12]) measuring the relationship between $\Pi(k)$ and $k$ can give insights on the future evolution of the network. This behaviour is easier to test on this data-set because the total number of new nodes that joined the network during the observed period is much higher than the number of nodes at the end of the interval, since many nodes join the network for a

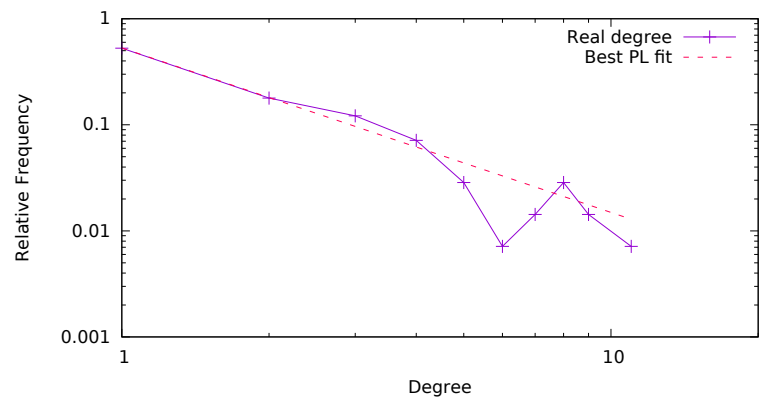

Fig. 3: The degree distribution for ninux, and the best powerlaw fit $x^{-\alpha}, \alpha=1.55$.

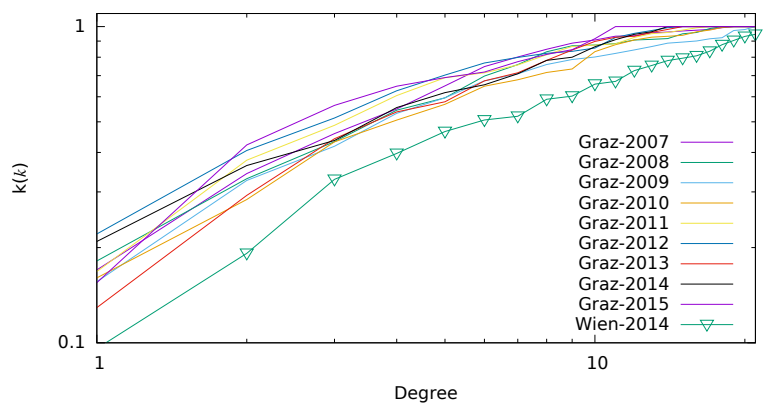

Fig. 4: The value of $\mathrm{k}(k)$ in the FFGraz and FFWien networks, separately computed per each year

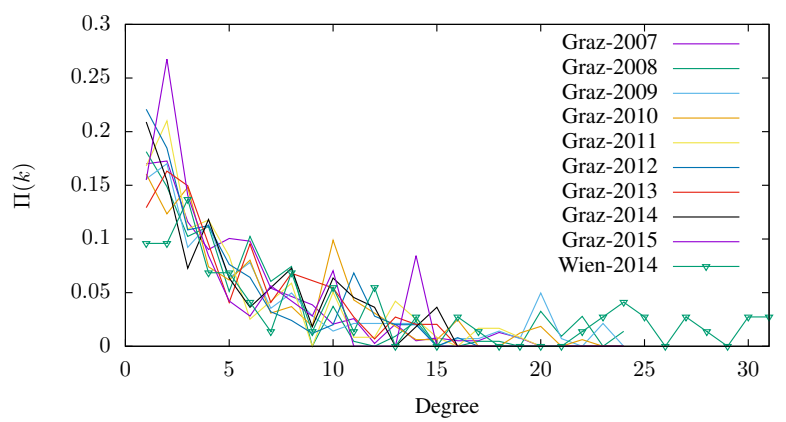

Fig. 5: The value of $\Pi(k)$ in the FFGraz and FFWien networks, separately computed per each year

limited period of time. To test the hypothesis of the preferential attachment model, for each year of the available data, for every new node added to the network the degree of the entry node was recorded and collected in a histogram that approximates $\Pi(k)$ (each node is counted only once at its first entry). To smooth fluctuations, as in [12], the cumulative function $\mathrm{k}(k)$ is considered:

$$
\mathrm{k}(k)=\int_{0}^{k} \Pi(x) d x
$$

In case $\Pi(k) \propto k$ then $\mathrm{k}(k) \propto 2$, which reported in a log-log graph should be a straight line of slope 2 .

Fig. 4 report $\mathrm{k}(k)$ for various years in the two FunkFeuer network and show clearly that there is no linear trend. Indeed, Fig. 5 confirms that for none of the years under analysis $\Pi(k)$ grows with $k$.

These results show that the two networks for which data is available (for the ninux network the monitoring period is too short) the growth model does not support the hypothesis of preferential attachment. As such, if the network keeps growing with the same model, coupled with the factual evidences explained in the next section, we do not expect the emergence of a scale-free behaviour. 


\section{A. Interpretation of the results}

Two features that influence the growth of a $\mathrm{CN}$ are: i) the limited range of wireless links ii) an upper bound on the number of incoming links.

Limited range: Wireless links are limited to a maximum length of about tens of kilometers and need to have line-ofsigh between the endpoints, while wired links do not have this limitation. Thus, a new node entering the network can not connect to any other node, and an existent node can acquire new links only from nodes placed at a distance smaller than the maximum range (which is not a fixed value and depends on a number of factors, such as the antenna type, the transmission power etc.). If the network grows in an urban area maintaining a constant density, hubs will be formed less likely than in a scale-free network.

Limited maximum node degree: A wireless node can be equipped with several physical radios, but more radios require more maintenance. Mounting tens of radios, cabling them, powering them, configuring them, is costly. While wireless ISPs use trellis and pay for the maintenance, a single person typically does not have the physical space, the resources and the time to install and maintain such a complex infrastructure. Thus, node degree can not grow indefinitely.

This result confirms and extends the analysis carried on portions of the Guifi network [7] that observed that some portions of Guifi did not show a scale-free behaviour. The authors suggest that this is true for networks that cover up to a certain geographical area and it is influenced by the degree of "planning" in the evolution of the network (planned or completely spontaneous). Another interpretation could be that Guifi, contrarily to the networks analysed in this paper is a real cooperative ISP, thus its mission is to bring Internet to the people. This probably leads to shorten as much as possible the path to the closest gateway, and a quasi-hierarchical network design is more suitable for this task. Instead, networks that have local connectivity as a goal may follow a different evolution path; more research is needed to formulate a sound interpretation.

\section{SOCIO-TECHNICAL ANALYSIS OF NINUX}

The following data is taken from network dumps collected in 2014 and extends the publication [5], the next two subsections expose the results and the third one jointly comments them. Direct interaction with the community was necessary to give a qualitative interaction of the quantitative results.

\section{A. The Ownership of the ninux network}

Fig. 6 presents the number of nodes possessed by the top 20 ninux participants, ordered by nodes owned, referring to a snapshot of the network in which the maximum number of nodes were present (140). Over a total of 78 owners, one user possess $17 \%$ of the nodes and the top five people own $31 \%$ of the nodes, top 13 people own roughly $50 \%$ of the nodes, 61 people own just one node. If we exclude the first individual (that we call $P_{t o p}$ ), the distribution is not particularly skewed, reflecting the fact that the number of owned nodes is generally limited by the number of physical locations in the city to which

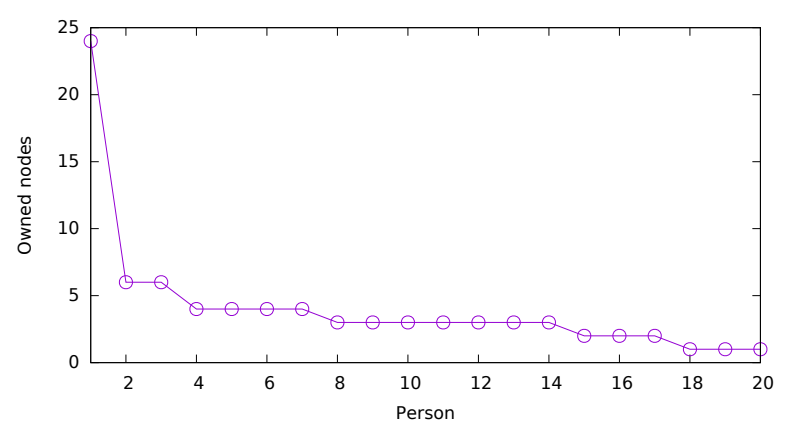

Fig. 6: The number of nodes per user in the ninux network, top 20 users.

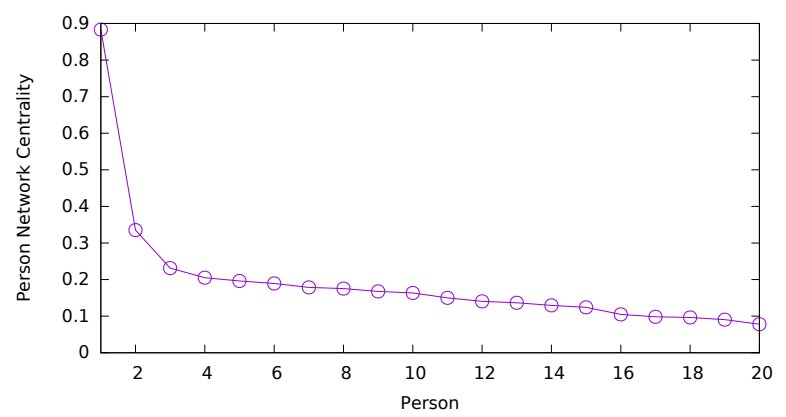

Fig. 7: The "person network centrality" for the participants to the ninux network, top 20 users.

the person has access (home, workplace, houses of relatives etc...). $P_{\text {top }}$ owns 24 nodes and is not the owner of all the locations where the nodes are placed, he is simply a technically skilled person that very often offers his help to set up the network for newcomers. As a result, he appears to be the owner and the technical manager of the nodes.

Fig. 7 shows the group betweenness centrality computed on all the nodes owned by the same person. The group betweenness centrality is the fraction of shortest paths that pass through at least one node in the group. Formally, if the network graph is a weighted graph $G(V, E)$, and $P_{i, j}=\left\{v_{i} \ldots v_{j}\right\}$ is the set of nodes that constitute the shortest path from node $v_{i}$ to node $v_{j}$ then the group centrality of a set of nodes $S=\left\{v_{1} \ldots v_{n}\right\} \subset V$ is given by:

$$
B(S)=\frac{\left\|\left\{P_{i, j} i, j \in(1 \ldots|V|) \mid S \cap P_{i, j} \neq \emptyset\right\}\right\|}{\left\|\left\{P_{i, j} i, j \in(1 \ldots|V|)\right\}\right\|}
$$

where $\|\cdot\|$ is the size of a set. The centrality metric is computed running Djikstra's algorithm on the weighted network topology, and, without information on the traffic matrix is the best estimation of the number of traffic flows that a group of nodes can intercept. Fig. 7 shows the "person network centrality", the ranked group centrality of the nodes owned by the same person and tells that $P_{t o p}$ can potentially control almost $90 \%$ of the traffic flows.

Figs. 6 and 7 outline a peculiar feature of a CN. As long as people are not allowed to own nodes out of their properties, 


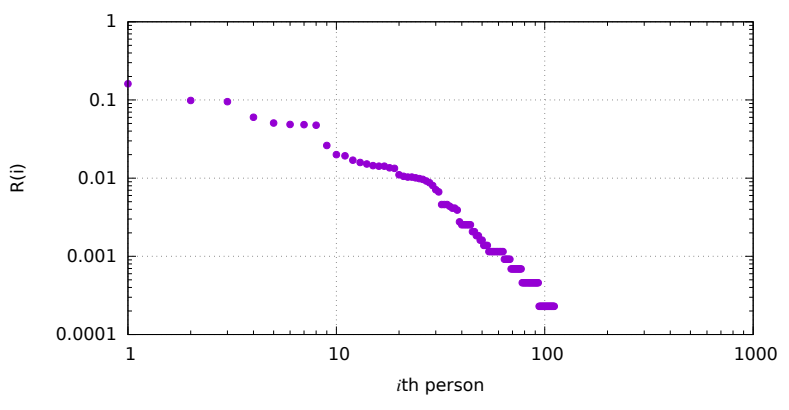

Fig. 8: The fraction of answered emails on the total in the mailing list.

the Wi-Fi range limitation does not allow a single person to be too central, and thus too critical for the network economy. This questions the peer-to-peer nature of the network in the case in which it is participated not only by individuals but also by associations, small business etc. that can be physically located in several places. In that case, the community must monitor the importance of those entities in order to avoid that any of them could become a single point of failure for the $\mathrm{CN}$, as $P_{\text {top }}$ is for ninux.

\section{B. The ninux mailing list}

The analysis of the mailing list messages helps understanding who are the individuals that lead the discussion inside the community. Two metrics defined in the literature have been chosen for this task [13]. The first is the normalized number of answered email per user: given a number $X$ of total messages that reply to some other message, and being $x_{i}$ the number of replies to a message sent by the $i$ th person, $R(i)=\frac{x_{i}}{X}$ is the relevance metric shown in Fig. 8. This is a basic metric that assumes that people that receive a high number of replies are able to generate interesting discussion topics, thus are considered important in the community.

Fig. 8 shows that the relevance to the mailing list is not equally distributed among the participants, a very small number of people lead the discussion. The cumulative distribution in Fig. 9 shows that as less as 6 people receive $50 \%$ of the overall answers.

The second metric is the centrality of a person in the mailing list social graph. The social graph is an undirected graph $G(V, E)$ in which every node $v_{i}$ is a person in the mailing list and there is an unweighted edge between two nodes $v_{i}, v_{j}$ if person $v_{j}$ ever answered to person $v_{i}$ (or vice-versa). Mailing list centrality is computed on the social graph for $v_{i}$ as in Eq. (2) when $S=\left\{v_{i}\right\}$. Betweenness centrality on mailing lists is used to understand who is able to make other people join the same discussion, so that he/she can facilitate the flow of information in the community. Again, Fig. 10 shows that there is a small number of people connecting all the other participants, and one in particular whose centrality is at least the double of the others.

Finally, Fig. 11 reports the percentage overlap on the two betweenness rankings from Fig. 7 and Fig. 10. The percentage

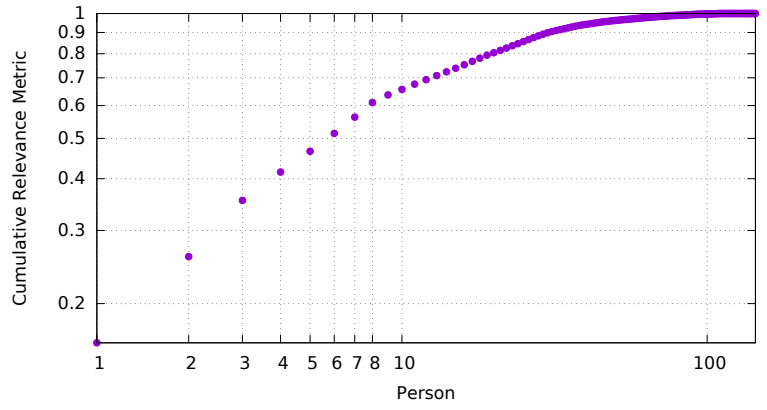

Fig. 9: The cumulative distribution of answered emails on the total in the mailing list.

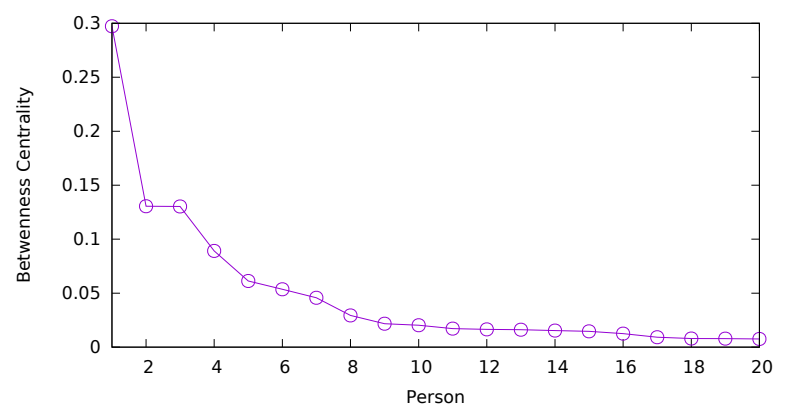

Fig. 10: The ranked centrality of the top 20 participants in the ninux mailing list.

overlap gives a measure of the correlation between the two rankings. Given a family of sets $B_{i}$ and the respective ordering functions $o_{i}(v)$ on their elements, we call $B_{i}^{k}$ the first $k$ element of $B_{i}$ ordered by $o_{i}(v): B_{i}^{k}=\left\{v \mid v \in B_{i}, o_{i}(v) \leq k\right\}$. Given two sets $B_{1}$ and $B_{2}$ the percentage overlap $p(k)$ is a function of $k$ that shows the percentage of elements present in both sets when considering only the first $k$ elements:

$$
p(k)=\frac{100}{k} \times\left\|B_{1}^{k} \cap B_{2}^{k}\right\|
$$

Fig. 11 shows two fundamental points: the first is that $P_{t o p}$, the person that owns more nodes and has the highest person network centrality is the same one that has the highest mailing list centrality. The second is that excluding the top person the correlation is not extremely strong, $p(10)=20 \%$ and $p(20)=$ $35 \%$.

\section{Interpretation of the results}

The distribution of the ownership, and thus the person centrality shows that, albeit the goal of the ninux community is to build a both technically and socially decentralized network, the results diverge from the goal. In 2014 one person in ninux managed a sufficient number of nodes to be able to control the network, and to represent a single point of failure. The same person, given his technical skills was a central person in the social network of the community, so he had an influential voice in the discussions. Indeed, direct discussion engaged 


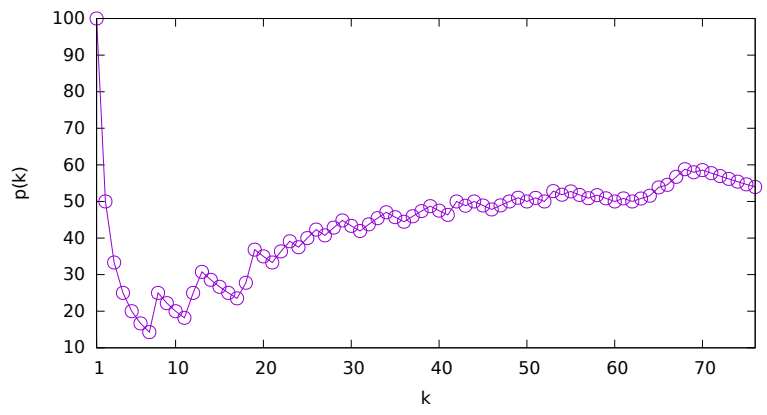

Fig. 11: The percentage overlap metric computed on the ranked mailing-list and group node centrality.

with people in the community revealed that this person left the community in 2015 and the nodes he managed started to fail and disconnect entire areas. At the time of writing the network is made of 87 nodes, 53 less than at its maximum expansion. In conclusion, the approach of the ninux community for the decentralization of the technical and social network was not successful since the network had a single point of failure represented by $P_{t o p}$.

However the situation changes excluding $P_{t o p}$ from the analysis. Fig. 6 shows that the maximum number of owned nodes is generally capped by the amount of physical locations that the users have access to, which intrinsically limits the chances of some individuals to take-over the network. Also, even if the social network metrics show that the relevance of the participants to the mailing list is not evenly distributed (this is indeed pretty common in many mailing list [14]) the correlation between the most relevant node owners and the most relevant members of the mailing list is low. This means that people participate to the community in diverse ways, with the construction of new nodes or through rising discussion topics.

\section{CONCLUSIONS}

This paper analyses the evolution of two large CNs and produces a socio-technical analysis of a third one. The analysis shows that wireless network with a bottom-up, peer-to-peer organization do not match a preferential attachment growth model. The emergence of hubs is reduced so the topology is less dependent on hubs compared to a scale-free network. More research, and time, is needed to tell if $\mathrm{CNs}$ will represent with their growth a different network model, or they will converge to some other known models, and what are the effects on the applications that a $\mathrm{CN}$ can sustain.

If the community networkers want to pursue their goal of building decentralized socio-technical infrastructure they will have to monitor the social interactions in the community, to verify that no single person or small group of people can take over the network. Metrics such as network centrality and person centrality, together with social network analysis as used in this paper represent a starting point to develop monitoring instruments that will give to the community the "pulse" of the network and decide if the community is following a direction that best represents the community's collective goal. This is particularly important when the network evolves and it becomes the interconnection not only between private people but also between for-profit activities, as it happens in the Guifi network. In that case, the $\mathrm{CN}$ must monitor that a single entity does not grow large enough or central enough to become the effective "owner" of the network, reducing the control power of the community on the infrastructure.

\section{REFERENCES}

[1] S. Jain and D. Agrawal, "Wireless community networks," Computer, vol. 36 , no. 8, pp. 90-92, 2003.

[2] J. Sderberg, "Free Space Optics in the Czech Wireless Community: Shedding Some Light on the Role of Normativity for User-Initiated Innovations," Science, Technology \& Human Values, vol. 36, no. 4, pp. 423-450, Jan. 2011

[3] S. Crabu, F. Giovanella, L. Maccari, and P. Magaudda, "A Transdisciplinary Gaze on Wireless Community Networks," TECNOSCIENZA: Italian Journal of Science \& Technology Studies, vol. 6, no. 2, pp. 113134, Jan. 2016

[4] L. Maccari, "An analysis of the Ninux wireless community network," in The Second International Workshop on Community Networks and Bottom-up-Broadband (CNBuB), 2013.

[5] L. Maccari and R. L. Cigno, "A week in the life of three large wireless community networks," Ad Hoc Networks, vol. 24, Part B, no. 0, pp. 175 - 190, 2015.

[6] L. Cerda-Alabern, "On the topology characterization of Guifi.net," in IEEE 8th International Conference on Wireless and Mobile Computing, Networking and Communications (WiMob), Oct. 2012.

[7] D. Vega, R. Baig, L. Cerd-Alabern, E. Medina, R. Meseguer, and L. Navarro, "A technological overview of the guifi.net community network," Computer Networks, vol. 93, pp. 260-278, 2015.

[8] C. Barz, C. Fuchs, J. Kirchhoff, J. Niewiejska, and H. Rogge, "OLSRv2 for Community Networks: Using Directional Airtime Metric with external radios," Computer Networks, vol. 93, Part 2, pp. 324-341, Dec. 2015.

[9] L. Cerda-Alabern, A. Neumann, and L. Maccari, "Experimental Evaluation of BMX6 Routing Metrics in a 802.11an Wireless-Community Mesh Network," in 3rd International Conference on Future Internet of Things and Cloud (FiCloud), Aug. 2015.

[10] R. Baig, R. Roca, L. Navarro, and F. Freitag, "Guifi.Net: A Network Infrastructure Commons," in Proceedings of the Seventh International Conference on Information and Communication Technologies and Development ACMDev. ACM, 2015.

[11] J. Kos, M. Milutinovic, and L. Cehovin, "nodewatcher: A substrate for growing your own community network," Computer Networks, vol. 93 , pp. 279-296, 2015.

[12] H. Jeong, Z. Nda, and A.-L. Barabsi, "Measuring preferential attachment in evolving networks," EPL (Europhysics Letters), vol. 61, no. 4, p. 567, 2003.

[13] C. Bird, A. Gourley, P. Devanbu, M. Gertz, and A. Swaminathan, "Mining Email Social Networks," in Proceedings of the 2006 International Workshop on Mining Software Repositories. ACM, 2006.

[14] S. L. Toral, M. R. Martnez-Torres, and F. Barrero, "Analysis of virtual communities supporting OSS projects using social network analysis," Information and Software Technology, vol. 52, no. 3, pp. 296-303, Mar. 2010. 